\title{
Erythromycin stimulates phasic gastric contractility as assessed with an isovolumetric intragastric balloon pressure measurement
}

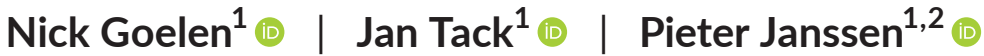

\author{
${ }^{1}$ Translational Research Center for \\ Gastrointestinal Disorders, KU Leuven, \\ Leuven, Belgium \\ ${ }^{2}$ VIPUN Medical, Mechelen, Belgium \\ Correspondence \\ Jan Tack, Translational Research Center \\ for Gastrointestinal Disorders, KU Leuven, \\ Herestraat 49, box 701, BE-3000 Leuven, \\ Belgium. \\ Email: Jan.tack@kuleuven.be \\ Funding information \\ VIPUN Medical; Fonds Wetenschappelijk \\ Onderzoek, Grant/Award Number: \\ $1 S 49317 \mathrm{~N}$
}

\begin{abstract}
Background: A novel technique to assess gastric motility by measuring the pressure in a low-volume intragastric balloon was developed to monitor (disordered) motility. We previously showed that this technique allows measuring pharmacologically induced inhibition of motility. In this study, we assessed whether it is possible to measure pharmacologically induced stimulation of gastric motility using $200 \mathrm{mg}$ erythromycin. Erythromycin is a highly effective stimulator of gastric emptying and contractility.

Methods: After an overnight fast, a nasogastric balloon catheter was introduced in healthy subjects. After inflation with $120 \mathrm{ml}$ of air, the catheter was connected to a pressure sensor. Intraballoon pressure was continuously recorded for $4 \mathrm{~h}$. After a baseline recording of $2 \mathrm{~h}, 200 \mathrm{mg}$ erythromycin was infused intravenously over $20 \mathrm{~min}$ while the recording continued for $2 \mathrm{~h}$. Epigastric symptoms were surveyed on 100-mm visual analogue scales. Motility was quantified from the pressure recording as a gastric balloon motility index. Wilcoxon signed-rank tests were performed. Data are shown as median (interquartile range).
\end{abstract}

Key Results: Six subjects were enrolled and five completed the procedures (age: 28 (25-29) years, body mass index: $\left.24.0(23.8-24.5) \mathrm{kg} \mathrm{m}^{-2}\right)$. One subject could not tolerate tube placement. Bloating, nausea, and epigastric sensation scores were 0 (0-3), 0 $(0-1)$, and $1(0-1) \mathrm{mm}$, respectively. Erythromycin significantly increased the motility index from $0.48(0.41-0.51)$ to $0.79(0.70-0.82)(p=0.03)$.

Conclusions and Inferences: Gastric motility assessed via pressure measurement in a low-volume intragastric balloon is able to detect pharmacologically stimulated motility in healthy subjects, which further validates this technique.

KEYWORDS

erythromycin, gastric motility, medical device, VIPUN Gastric Monitoring System

\section{1 | INTRODUCTION}

Gastric motility is a major driver of gastric emptying and is subject to complex neurohumoral control mechanisms. ${ }^{1}$ Bedside techniques to accurately assess gastric motility available for clinical care are scarce. We developed the VIPUN ${ }^{\text {TM }}$ Gastric Monitoring System (VIPUN GMS;
KU Leuven) to provide a continuous real-time indicator of gastric motility at the patient's bedside. The technique incorporates a doublelumen nasogastric feeding tube with integrated intragastric balloon at the distal tip. One lumen provides enteral access, and the second lumen is used to inflate the balloon with a fixed volume of air and to measure intraballoon pressure. The system provides a quantitative 
motility index. We previously demonstrated that the technique is able to detect codeine-induced inhibition of motility in healthy subjects. ${ }^{2}$ To complement that validation study, we set out to demonstrate that the technique can also detect pharmacologically enhanced gastric motility by means of erythromycin administration.

Erythromycin is a macrolide antibiotic with a highly effective gastroprokinetic effect as a motilin receptor agonist via facilitation of cholinergic activity. ${ }^{3}$ Erythromycin at a dose of $40 \mathrm{mg}$ induces phase III-like contractility in health and disease. This effect can be suppressed by atropine. ${ }^{4}$ On the other hand, 200 mg erythromycin induces prolonged phasic antral contractions shortly after infusion. ${ }^{4,5}$ At $200 \mathrm{mg}$, the effect is atropine-resistant and probably mediated by direct action on the muscle. ${ }^{4}$ The dose-dependent involvement of different pathways can affect drug efficacy and safety. ${ }^{3}$ These doses are substantially lower than normally used for antibiotic purposes and make it an attractive pharmacological agent for mechanistic studies. $^{3,6}$ We hypothesized that the GMS can detect the stimulatory effect of erythromycin on motility.

\section{2 | MATERIALS AND METHODS}

The investigation was approved by the Ethics Committee Research UZ/KU Leuven (reference: S62862) and the Belgian competent authorities (AFMPS/80M0783) and performed in accordance with the Declaration of Helsinki.

\section{1 | Investigational medical device}

A 12 French nasogastric double-lumen feeding tube with a polyurethane balloon (Via Biomedical) attached at the distal end was used (Figure 1). One lumen was used to inflate/deflate the balloon and to measure the intraballoon pressure. The proximal end of this lumen was connected to a pressure sensor (MPX2050DP, NXP Freescale ${ }^{\mathrm{TM}}$ ) and data acquisition system DI-245, DATAQ ${ }^{T M}$ Instruments) outside of the body. This setup, also referred to as the GMS (KU Leuven), has been described previously in detail. ${ }^{2,7}$ Pressure data were collected at $5 \mathrm{~Hz}$ with a resolution of $0.1 \mathrm{mmHg}$ and analyzed offline by a custom-made algorithm after completion of the study visit.

\section{2 | Study design}

This was a single center, investigation in six healthy adults. Subjects provided written informed consent prior to screening and other study-related procedures.

\section{3 | Study population}

Healthy adults with a body mass index between 18 and 30 without gastrointestinal symptoms or known contra-indications for

\section{Key Message}

A new technique to quantify gastric contractility via pressure measurement in a low-volume intragastric balloon, the VIPUN Gastric Monitoring System, was developed. It was previously shown that the technique is able to differentiate physiologic and pharmacologically-inhibited contractility. The current study further contributes to the validation of this technique. We demonstrated that the isovolumetric balloon measurement was also able to detect the stimulating effect of $200 \mathrm{mg}$ erythromycin in a cohort of healthy adults.

nasogastric tube placement or erythromycin administration were eligible for enrollment. Chronic dyspeptic symptoms were assessed by means of the validated patient assessment of upper gastrointestinal symptom severity index questionnaire (PAGI-SYM). ${ }^{8}$

Pregnancy was excluded at the start of the study visit by means of a urine pregnancy test. Electrocardiograms were taken to exclude QT-prolongation ( $\geq 400 \mathrm{~ms}$ ) during screening and during erythromycin administration. Subjects were instructed to remain fasted for $12 \mathrm{~h}$ and to refrain from alcohol, caffeine, and medication $12 \mathrm{~h}$ prior to the study visit.

\section{4 | Study procedures}

The catheter was placed with the balloon in the stomach via the nasogastric route. Once positioned, the balloon was inflated with $120 \mathrm{ml}$ air. Intraballoon pressure was recorded continuously for 4 h. After 2 h, 200 mg erythromycin (Erythrocine I.V., $1 \mathrm{mg} \mathrm{ml}^{-1}$, Amdipharm UK Limited) was infused intravenously over a 20-min period. Visual analogue scales (VAS, 100 mm, 0: absent, 100: worst possible sensation) for hunger, satiation, upper-abdominal bloating, nausea, and epigastric sensation/pain (pain threshold: $50 \mathrm{~mm}$ ) were completed prior to catheter placement and every $15 \mathrm{~min}$ thereafter. After $4 \mathrm{~h}$ of recording, the balloon was deflated and the catheter removed.

\section{5 | Analysis}

Intraballoon pressure variations were analyzed by a custom-made algorithm in Matlab ((MatLab ${ }^{\circledR}$ R2018a, The MathWorks ${ }^{\circledR}$ ) as published earlier. ${ }^{2}$ Data processing and cleaning included the removal of cardiac, respiratory, and movement artifacts from the signal. Pressure peaks induced by gastric contractions were identified based on the peak frequency ( $<6$ contractions per min), a minimum inter-peak distance of $17 \mathrm{~s}$, a minimum peak base width of $17 \mathrm{~s}$, and a minimum half-prominence width of $7 \mathrm{~s}$. These parameters were defined empirically based on a training data set by experts on the 


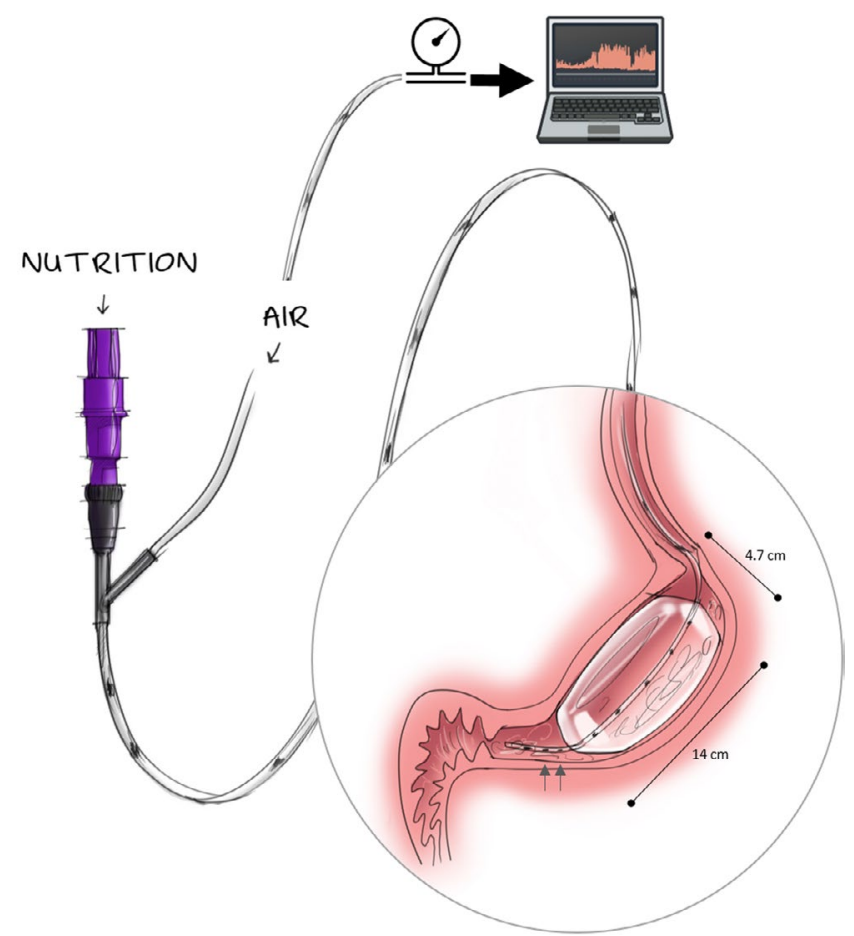

FIGURE 1 The gastric monitoring system consists of a 12-French double-lumen nasogastric balloon catheter and a control unit recording pressure fluctuations. One lumen connects an inflatable balloon to the control unit, the other lumen provides enteral access (gray arrows). Artist's impression of dimensions. Adapted with permission from Goelen et $\mathrm{al}^{2}$

interpretation of gastric motility. The parameters were fixed prior to this and previous studies. ${ }^{2}$ A Gastric Balloon Motility Index (GBMI) was calculated as the fraction of time during which gastric contractions were detected ranging from 0 (no contraction) to 1 (contractions continuously present). ${ }^{2}$

Data were summarized by following three periods for statistical analysis: a baseline period of $120 \mathrm{~min}$, a 20 -min period during erythromycin administration and a post-infusion period of $100 \mathrm{~min}$.

Data were analyzed by means of Wilcoxon signed-rank tests (SAS University edition 9.4, SAS Institute Inc.). It was hypothesized that GBMI is higher in the period during erythromycin infusion relative to baseline. Hence, a one-sided test was performed. A significance level of .05 was applied without correction for multiple testing. Results are presented as median (interquartile range).

\section{RESULTS}

Nine subjects were screened of which two were excluded due to a prolonged QT-interval and one due to chronic dyspeptic symptoms based on the PAGI-SYM questionnaire (Figure 2). Hence, 6 subjects were enrolled, of which one subject could not tolerate the catheter placement procedure and withdrew consent. The per protocol population comprises data from 5 subjects which are presented here.

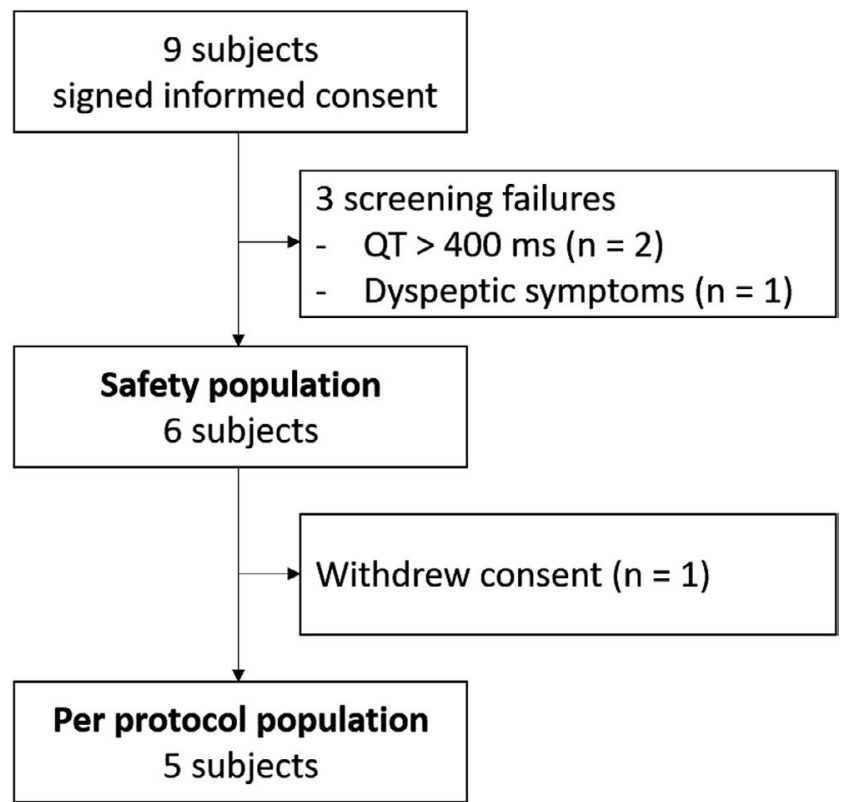

FIGURE 2 Subject disposition. Reasons for exclusion from the per protocol population are shown.

The median age was 28 (25-29) years, and median body mass index was $24.0(23.8-24.5) \mathrm{kg} \mathrm{m}^{-2}$. The median GBMI at baseline was 0.48 (0.41-0.51). Erythromycin administration resulted in a significant increase in motility (GBMI $=0.79$ (0.70-0.82), one-sided $p=0.0313$ ) (Figures 3 and 4). Motility increased numerically for all subjects within 20 min following erythromycin administration (Figure 5). Motility remained elevated relative to baseline in the post-infusion period $(0.84(0.80-0.84)$, one-sided $p=0.0313)$.

The median symptom score was $0(0-3) \mathrm{mm}$ for bloating, $0(0-1)$ $\mathrm{mm}$ for nausea, and $1(0-1) \mathrm{mm}$ for epigastric sensation. The maximum observed symptom scores at a single point in time were $30 \mathrm{~mm}$ for bloating, $75 \mathrm{~mm}$ for nausea, and $30 \mathrm{~mm}$ for sensation. The sensation/pain threshold, a priori set at $50 \mathrm{~mm}$, was not reached. Hunger, satiation, and epigastric symptom scores are shown in Figure 6. Erythromycin administration did not significantly affect hunger, satiation, or epigastric symptom scores, although maximum epigastric symptom scores were reported during or shortly after erythromycin infusion.

\section{4 | DISCUSSION}

This study aimed to demonstrate that erythromycin-enhanced gastric motility can be detected by means of a new isovolumetric balloon catheter-based technique in healthy subjects. Motility was quantified as a motility index based on intraballoon pressure fluctuation and offline peak detection.

Six subjects were exposed to the investigational device. One subject could not tolerate the placement procedure of the nasogastric tube due to continuous retching and withdrew consent. Retching was an anticipated adverse device effect, as is the case for any standard nasogastric feeding tube. ${ }^{9}$ No other untoward events were observed. From other studies, ${ }^{7}$ we know that the balloon, with a length 


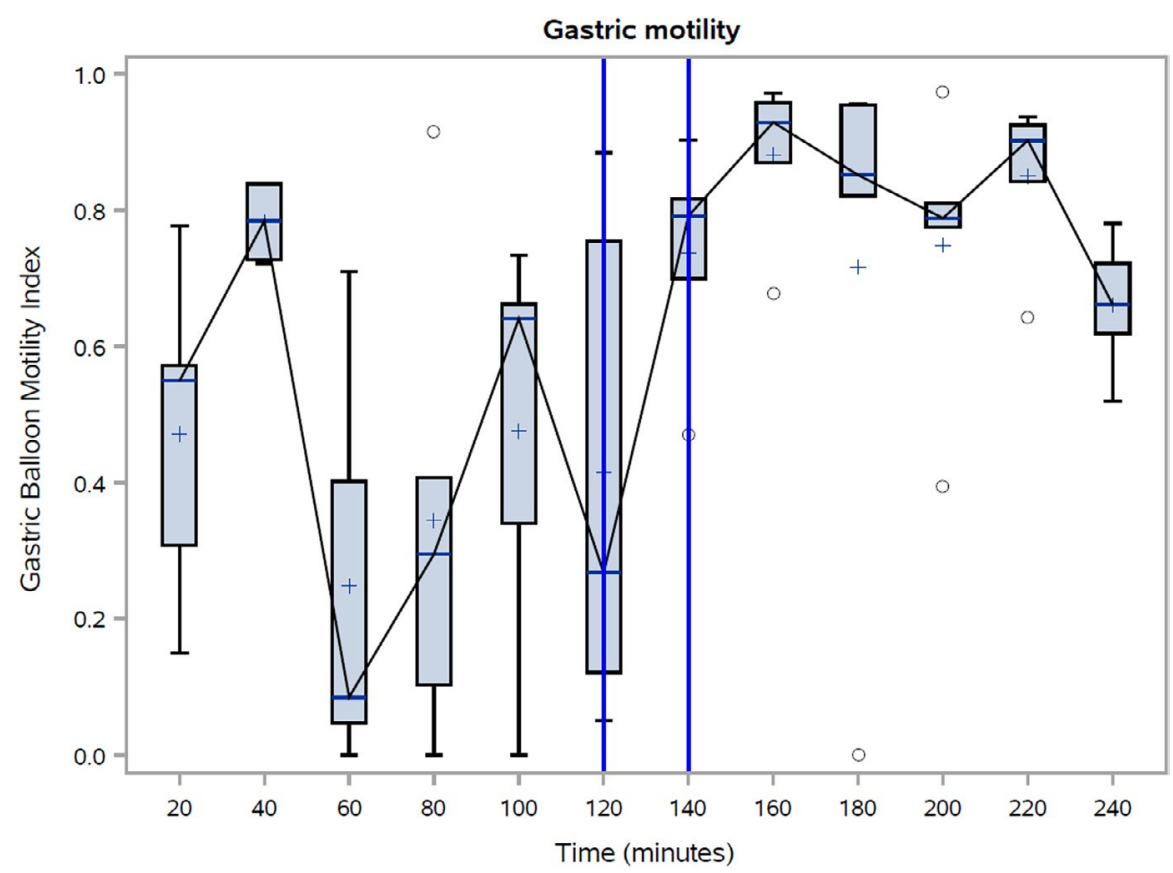

FIGURE 3 Boxplots of gastric balloon motility index (GBMI) averaged per 20 min. Erythromycin was infused intravenously in the period $t=120-140 \mathrm{~min}$ (blue vertical lines). Box and whiskers indicate median, quartile ranges, and minimum and maximum observations that are not outliers. Outliers are shown as circles; mean is indicated as +

\section{Gastric motility}

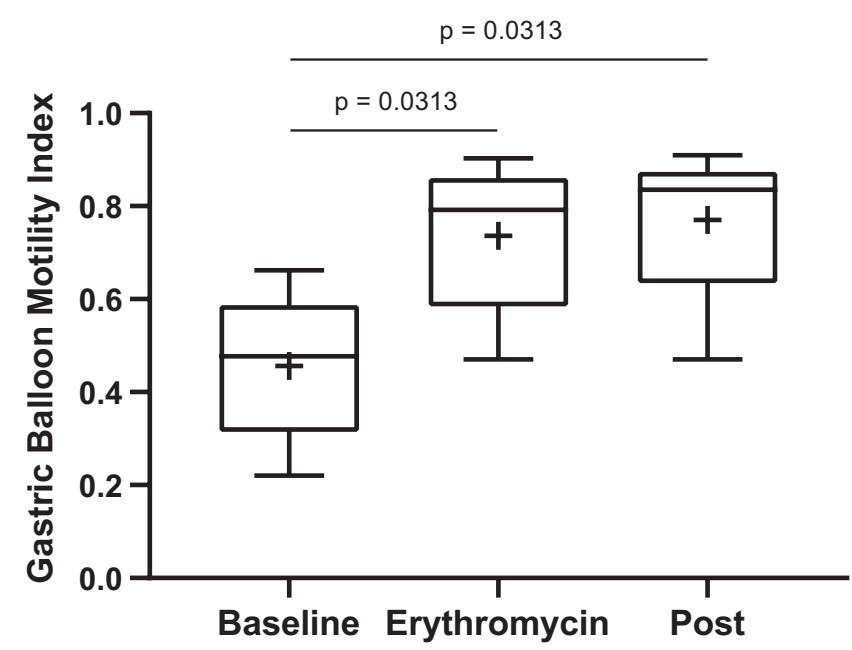

FIG URE 4 Boxplots of Gastric Balloon Motility Index (GBMI) at baseline, during 20-min erythromycin infusion and during the 100-min post-infusion period. Mean is indicated as + . One-sided $p$ values obtained by Wilcoxon signed-rank test are shown

of $14 \mathrm{~cm}$, is typically positioned in the corpus and antrum, although this was not confirmed radiographically in this study.

Enteral feeding intolerance is a frequent complication encountered at the intensive care unit. Enteral feeding intolerance may result in vomiting and failure to reach nutritional targets. ${ }^{10-12}$ Objective reliable bedside tools to monitor (in-)tolerance to enteral nutrition are lacking. We developed the GMS to address this need for an indicator of gastric motor function in critically ill patients. A

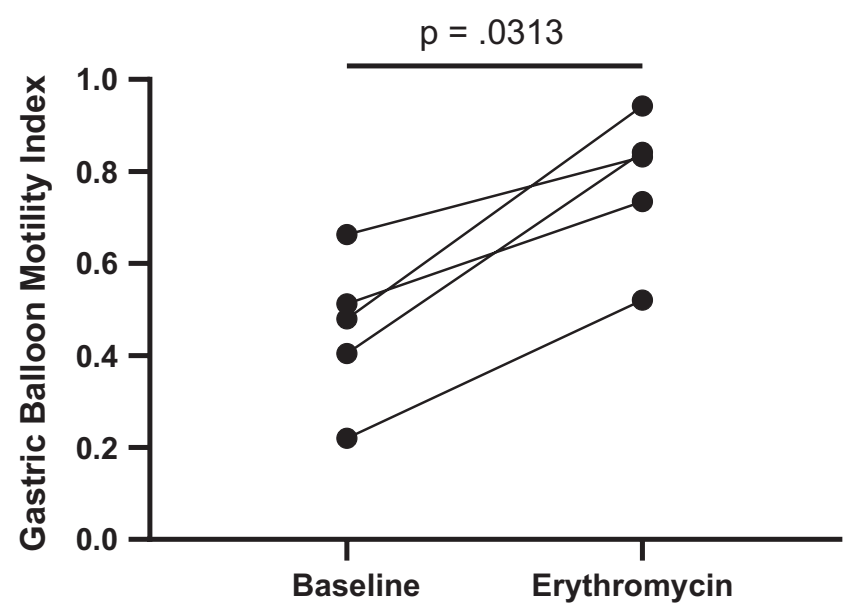

FIGURE 5 Before-after plot of erythromycin-induced changes in Gastric Balloon Motility Index (GBMI) per subject. One-side $p$ value obtained by Wilcoxon signed-rank test

real-time readout of motility can offer feedback to the clinician on the efficacy of the administered therapy, be it nutritional or pharmacological. Erythromycin is used empirically and off-label to promote gastric function in critically ill patients. ${ }^{13}$

GBMI during erythromycin infusion was significantly higher compared to baseline. GBMI increased within 20 min after initiation of erythromycin infusion, without exception. It is well known that erythromycin stimulates gastric motility at a dose of $200 \mathrm{mg}$. Thus, this finding confirms our primary hypothesis that a low-volume intragastric balloon can be used to detect and quantify stimulated gastric motility. ${ }^{4,5}$ The rise in motility index can be attributed to 


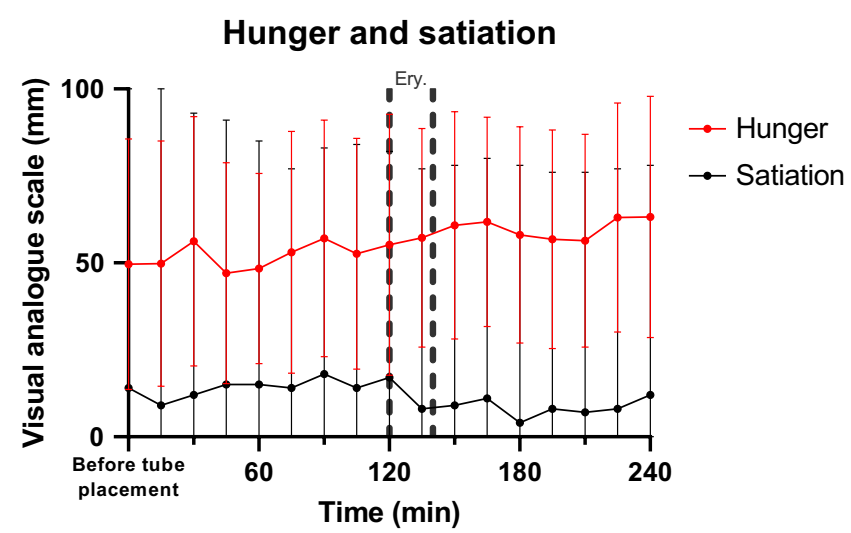

Epigastric symptom scores

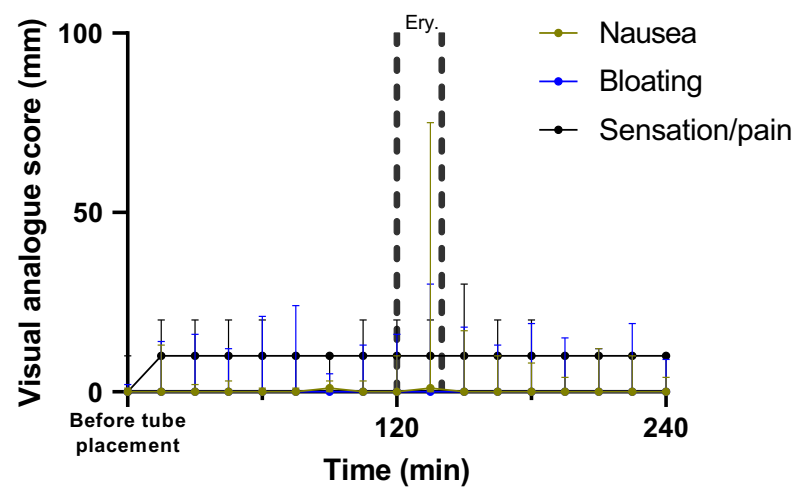

FIGURE 6 Hunger, satiation and epigastric symptom scores. Median and range (minimum to maximum) of absolute visual analogue scale scores $(\mathrm{mm})$ are shown. First data point was measured prior to placement of nasogastric tube. 20-min erythromycin (Ery.) infusion is indicated by vertical dashed lines. Pain threshold on epigastric sensation scale was set at 50/100 mm

the induction of phase III-like contractility patterns in combination with random contractions similar to phase II of the migrating motor complex. ${ }^{5}$ This prokinetic effect also accelerates delayed gastric emptying rate in patients with gastroparesis, functional dyspepsia and enteral feeding intolerance in critically ill patients. ${ }^{12-23}$ The relatively high motility observed during the baseline period at $t=40 \mathrm{~min}$ can be attributed to the spontaneous occurrence of phase III in two subjects (Figure 5).

Epigastric sensations or symptoms were mild or absent. Although erythromycin administration provoked a burst of high-amplitude contractions, we did not observe a simultaneous rise in hunger sensation; therefore, the term phase III-like contractions was used. ${ }^{5}$ Spontaneous physiologic phase III fronts are associated in time with a rise in hunger and plasma concentrations of motilin and ghrelin. Indeed, our findings contrast that paradigm and are in contrast with a study by Tack et al. that reported that $40 \mathrm{mg}$ erythromycin-induced phase III activity and increased hunger scores. ${ }^{24}$ On the other hand, sustained stimulation of contractile activity using the cholinesterase inhibitor neostigmine was not associated with a rise in hunger sensations. ${ }^{24}$ The erythromycin-induced contractions in the present study are reminiscent of the latter motility pattern, which may explain the lack of a hunger peak.

Limitations of this investigation include the limited sample size, substantial variation in motility patterns during baseline, lack of placebo-control, and lack of an external reference measure. All four concerns are in part mitigated by the well-described robust effect of erythromycin to evoke bursts of high-amplitude contractions. ${ }^{4}$ Moreover, each subject served as its own control. This robust model allowed us to demonstrate that erythromycin administration consistently resulted in a rise in motility index. Statistical significance was reached in merely five subjects using a one-sided test. Post hoc power analysis confirmed adequacy of a sample of four subjects to reach a power of $80 \%$. Type II errors cannot be excluded with regard to the secondary endpoints.

The current observations supplement earlier work where we demonstrated that the GMS can detect codeine-inhibited contractility. This inhibition was correlated with delay in gastric emptying. Gastric emptying was not assessed in the present study, but the acceleration of gastric emptying by 200 mg erythromycin was convincingly and repetitively documented in the literature. ${ }^{4,19,20,25-29}$ Taken together, these two pharmacological models contribute to the validation of the GMS as quantitative indicator of phasic gastric contractility underlying gastric emptying.

Even though this study is limited by its sample size, it is possible to conclude that the VIPUN GMS is able to detect pharmacologically enhanced phasic gastric contractility. This investigation contributes to the indirect validation of the technique as a promising tool to monitor gastric motility.

\section{ACKNOWLEDGEMENTS}

This study was registered on Clinicaltrials.gov (NCT04066231). We would like to thank Annelies Geeraerts, Jolien Schol, Hannelore Geysen, Lien Timmermans, and Carla Baeken for their assistance during the experiments. NG is a SB PhD fellow of the Research Foundation-Flanders (FWO, grant number: 1S49317N). All authors were involved in the conceptualization of the study and interpretation of the results; NG designed and performed the experiments, analyzed the data, and drafted the manuscript; PJ and JT designed the study and critically revised the manuscript.

\section{CONFLICT OF INTEREST}

NG and PJ own shares in VIPUN Medical. JT received a research grant from VIPUN Medical to support this academic investigatorinitiated study.

\section{ORCID}

Nick Goelen (D) https://orcid.org/0000-0001-7812-1740

Jan Tack (D) https://orcid.org/0000-0002-3206-6704

Pieter Janssen (D) https://orcid.org/0000-0003-1792-953X

\section{REFERENCES}

1. Hansen MB. Neurohumoral control of gastrointestinal motility. Physiol Res. 2003;52(1):1-30. 
2. Goelen N, de Hoon J, Morales JF, et al. Codeine delays gastric emptying through inhibition of gastric motility as assessed with a novel diagnostic intragastric balloon catheter. Neurogastroenterol Motil. 2020;32(1):e13733.

3. Broad JMS, Samadi M, Martin JE, Dukes GE, Sanger GJ. Regionaland agonist-dependent facilitation of human neurogastrointestinal functions by motilin receptor agonists. $\mathrm{Br} J$ Pharmacol. 2012;167(4):763-774.

4. Coulie B, Tack J, Peeters T, Janssens J. Involvement of two different pathways in the motor effects of erythromycin on the gastric antrum in humans. Gut. 1998;43(3):395-400.

5. Bjornsson ES, Abrahamsson H. Comparison between physiologic and erythromycin-induced interdigestive motility. Scand $J$ Gastroenterol. 1995;30(2):139-145.

6. Patcharatrakul T, Gonlachanvit S. Technique of functional and motility test: how to perform antroduodenal manometry. J Neurogastroenterol Motil. 2013;19(3):395-404.

7. Goelen N, Doperé G, Morales J, et al.Enteral nutrition dose- dependently inhibits phasic gastric motility. European Society of Neurogastroenterology and Motility meeting; 2019 September 5-7, 2019; Lisbon.

8. Kindt S, Dubois D, Van Oudenhove L, et al. Relationship between symptom pattern, assessed by the PAGI-SYM questionnaire, and gastric sensorimotor dysfunction in functional dyspepsia. Neurogastroenterol Motil. 2009;21(11):1183-e105.

9. Singer AJ, Konia N. Comparison of topical anesthetics and vasoconstrictors vs lubricants prior to nasogastric intubation: a randomized, controlled trial. Acad Emerg Med. 1999;6(3):184-190.

10. Tatsumi H. Enteral tolerance in critically ill patients. J Intensive Care. 2019;7:30.

11. Chapman M, Fraser R, Vozzo R, et al. Antro-pyloro-duodenal motor responses to gastric and duodenal nutrient in critically ill patients. Gut. 2005;54(10):1384-1390.

12. Chapman MJ, Deane AM. Gastrointestinal dysfunction relating to the provision of nutrition in the critically ill. Curr Opin Clin Nutr Metab Care. 2015;18(2):207-212.

13. Deane AM, Chapman MJ, Reintam Blaser A, McClave SA, Emmanuel A. Pathophysiology and treatment of gastrointestinal motility disorders in the acutely III. Nutr Clin Pract. 2019;34(1):23-36.

14. Ritz MA, Chapman MJ, Fraser RJ, et al. Erythromycin dose of $70 \mathrm{mg}$ accelerates gastric emptying as effectively as $200 \mathrm{mg}$ in the critically ill. Intensive Care Med. 2005;31(7):949-954.

15. Chapman MJ, Deane AM, O'Connor SL, et al. The effect of camicinal (GSK962040), a motilin agonist, on gastric emptying and glucose absorption in feed-intolerant critically ill patients: a randomized, blinded, placebo-controlled, clinical trial. Crit Care. 2016;20(1):232.

16. Larson JM, Tavakkoli A, Drane WE, Toskes PP, Moshiree B. Advantages of azithromycin over erythromycin in improving the gastric emptying half-time in adult patients with gastroparesis. J Neurogastroenterol Motil. 2010;16(4):407-413.
17. Moshiree B, McDonald R, Hou W, Toskes PP. Comparison of the effect of azithromycin versus erythromycin on antroduodenal pressure profiles of patients with chronic functional gastrointestinal pain and gastroparesis. Dig Dis Sci. 2010;55(3):675-683.

18. Thor P, Lorens K, Tabor S, et al. Dysfunction in gastric myoelectric and motor activity in Helicobacter pylori positive gastritis patients with non-ulcer dyspesia. J Physiol Pharmacol. 1996;47(3):469-476.

19. Arts J, Caenepeel P, Verbeke K, Tack J. Influence of erythromycin on gastric emptying and meal related symptoms in functional dyspepsia with delayed gastric emptying. Gut. 2005;54(4):455-460.

20. Janssens J, Peeters TL, Vantrappen G, et al. Improvement of gastric emptying in diabetic gastroparesis by erythromycin - Preliminary studies. N Engl J Med. 1990;322(15):1028-1031.

21. Annese V, Janssens J, Vantrappen $G$, et al. Erythromycin accelerates gastric emptying by inducing antral contractions and improved gastroduodenal coordination. Gastroenterology. 1992;102(3):823-828.

22. Camilleri M, Chedid V, Ford AC, et al. Gastroparesis. Nat Rev Dis Primers. 2018;4(1):41.

23. Tack J, Camilleri M. New developments in the treatment of gastroparesis and functional dyspepsia. Curr Opin Pharmacol. 2018;43:111-117.

24. Tack J, Deloose E, Ang D, et al. Motilin-induced gastric contractions signal hunger in man. Gut. 2016;65(2):214-224.

25. Chapman MJ, Fraser RJ, Kluger MT, Buist MD, De Nichilo DJ. Erythromycin improves gastric emptying in critically ill patients intolerant of nasogastric feeding. Crit Care Med. 2000;28(7):2334-2337.

26. Ramirez B, Eaker EY, Drane WE, Hocking MP, Sninsky CA. Erythromycin enhances gastric emptying in patients with gastroparesis after vagotomy and antrectomy. Dig Dis Sci. 1994;39(11):2295-2300.

27. Petrakis IE, Vrachassotakis N, Vassilakis SJ, Sciacca V, Chalkiadakis G. Erythromycin enhances solid-phase gastric emptying in induced-hyperglycemia in patients with truncal vagotomy and pyloroplasty. Dig Dis Sci. 2000;45(5):937-945.

28. Burt M, Scott A, Williard WC, et al. Erythromycin stimulates gastric emptying after esophagectomy with gastric replacement: a randomized clinical trial. J Thorac Cardiovasc Surg. 1996;111(3):649-654.

29. Maes BD, Hiele MI, Geypens BJ, et al. Pharmacological modulation of gastric emptying rate of solids as measured by the carbon labelled octanoic acid breath test: influence of erythromycin and propantheline. Gut. 1994;35(3):333-337.

How to cite this article: Goelen N, Tack J, Janssen P.

Erythromycin stimulates phasic gastric contractility as assessed with an isovolumetric intragastric balloon pressure measurement. Neurogastroenterology \& Motility.

2020;00:e13991. https://doi.org/10.1111/nmo.13991 